\title{
Gaussian and Non-Gaussian Distributions of Fracture Properties in Tensile Stretching of High-Density Polyethylene
}

Tao Liao ${ }^{1}$, Xiao Yang ${ }^{1,2}$, Xintong Zhao ${ }^{1,2}$,Yujing Tang ${ }^{3}$, Zhiyong Jiang ${ }^{1 *}$, Yongfeng $\operatorname{Men}^{1,2 *}$

${ }^{1}$ State Key Laboratory of Polymer Physics and Chemistry, Changchun Institute of Applied Chemistry, Chinese Academy of Sciences, Renmin Street 5625, Changchun 130022, China

${ }^{2}$ University of Science and Technology of China, Hefei 230026, China

${ }^{3}$ Sinopec Beijing Research Institute of Chemical Industry, Beijing 100013, China

Corresponding E-mail address:jiangzhy@ciac.ac.cn (Z. J.), men@ciac.ac.cn (Y. M.) 

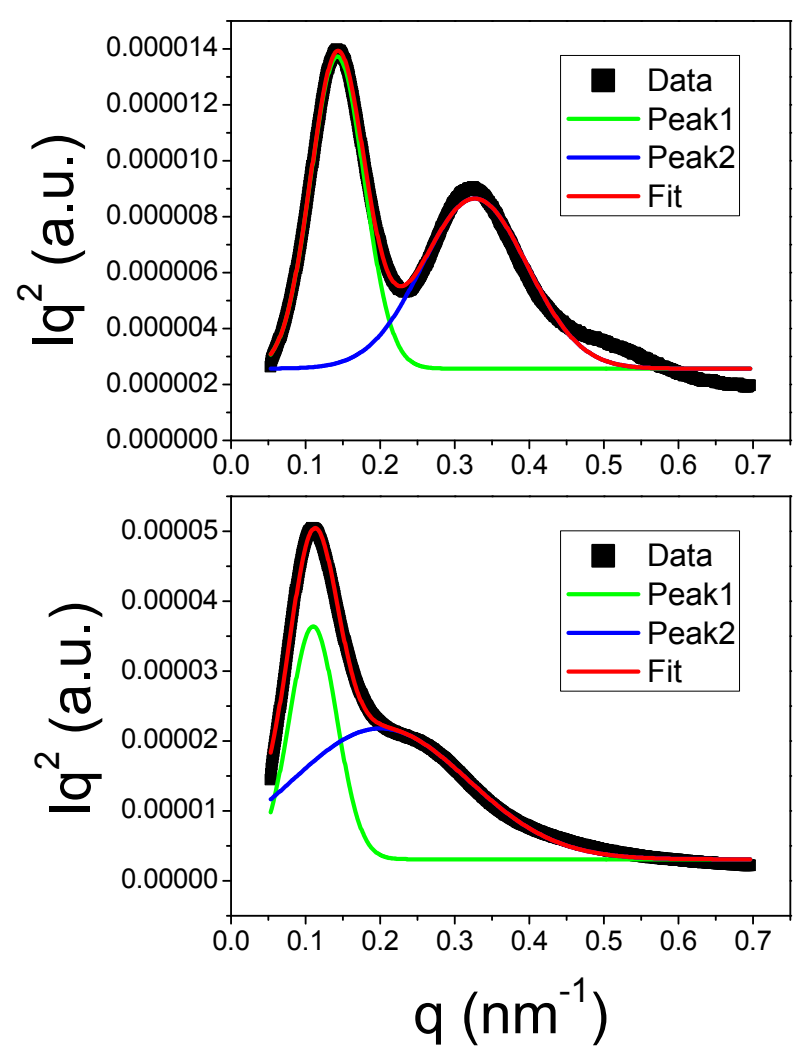

Figure S1. Decomposition of one-dimensional scattering intensity distribution profiles into contributions of the two lamellar stacks for the isothermally crystallized samples of HDPE-151K (top) and HDPE-932K (bottom) using two Gaussian functions. 


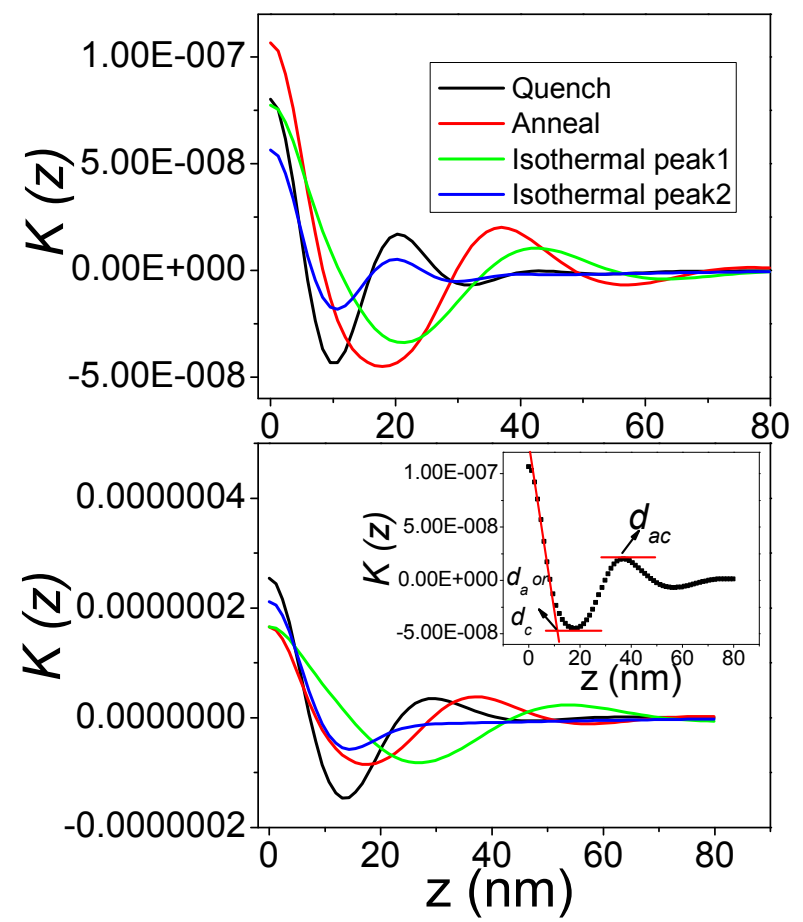

Figure S2. Correlation functions $K(z)$ for HDPE-151K (top) and HDPE-932K (bottom) samples. The inset of the bottom plot shows how the long period $\left(d_{a c}\right)$ together with the average thickness of crystalline lamellae $\left(d_{c}\right)$ and amorphous layers $\left(d_{a}\right)$ are determined. The smaller value is assigned to $d_{a}$ or $d_{c}$ depending on the crystallinity. 

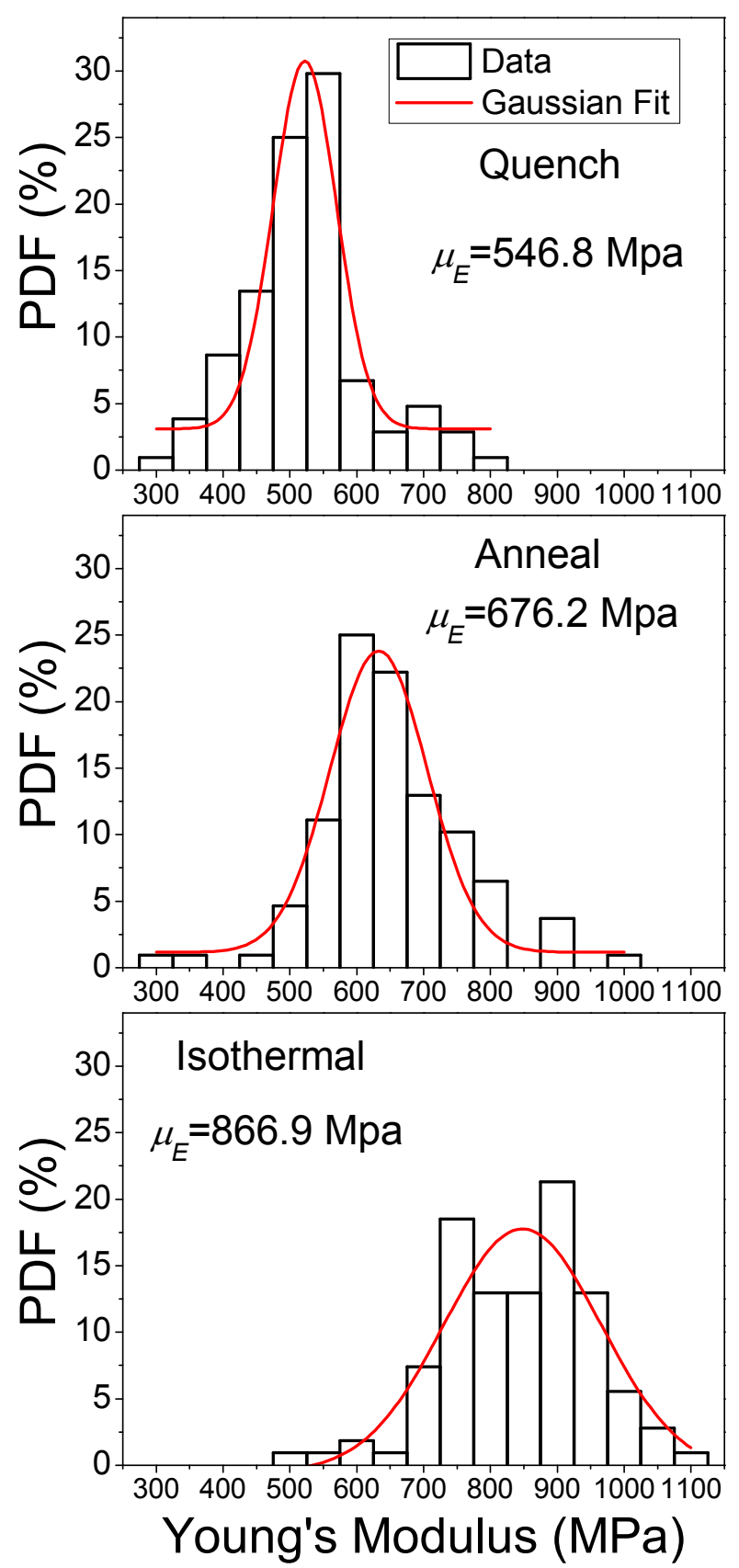

Figure S3. Probability density functions of the Young's modulus for HDPE-151K (from top to bottom: quenched, annealed, and isothermally crystallized samples). The average value of Young's modulus is indicated in the inset of the figure. 

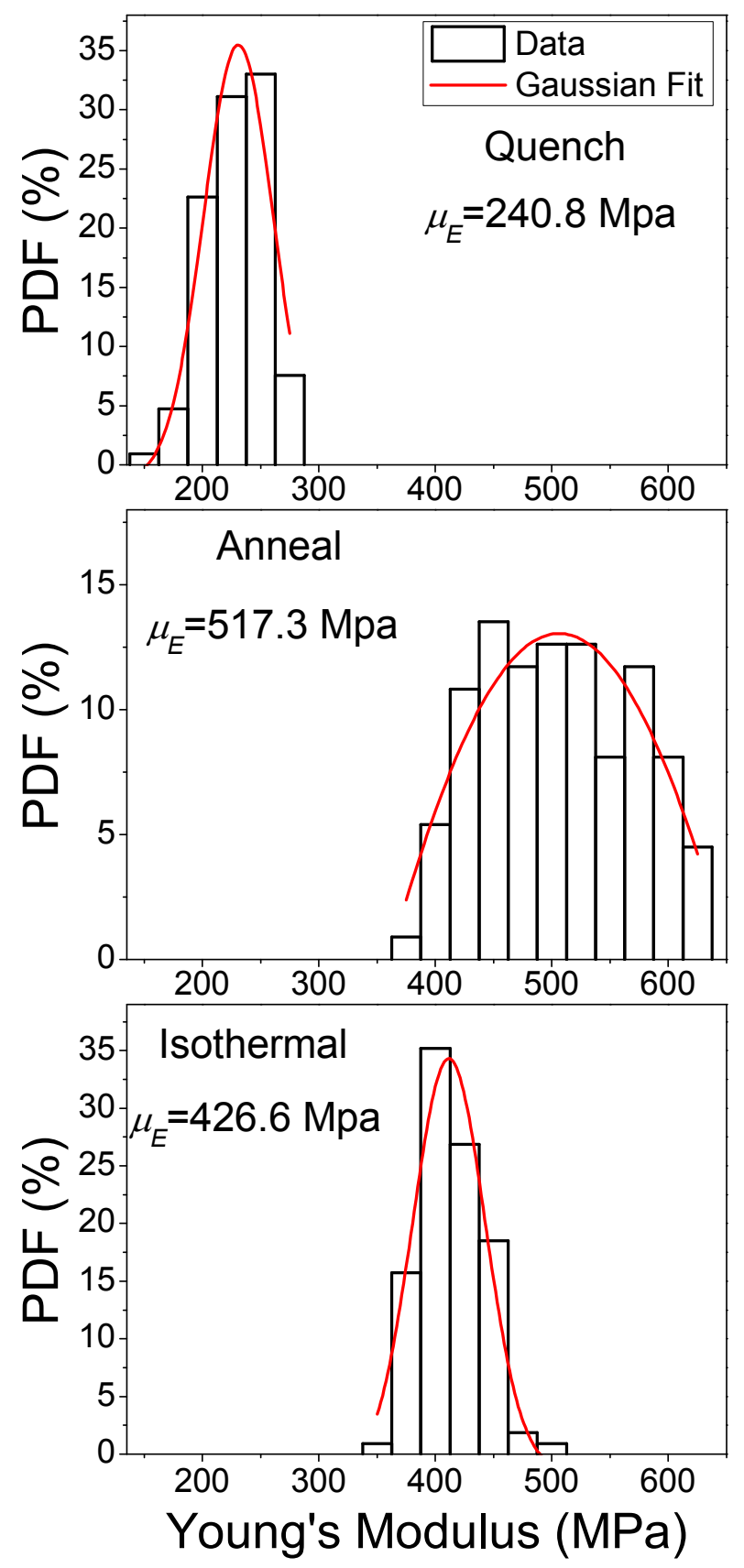

Figure S4. Probability density functions of the Young's modulus for HDPE-932K (from top to bottom: quenched, annealed, and isothermally crystallized samples). The average value of Young's modulus is indicated in the inset of the figure. 


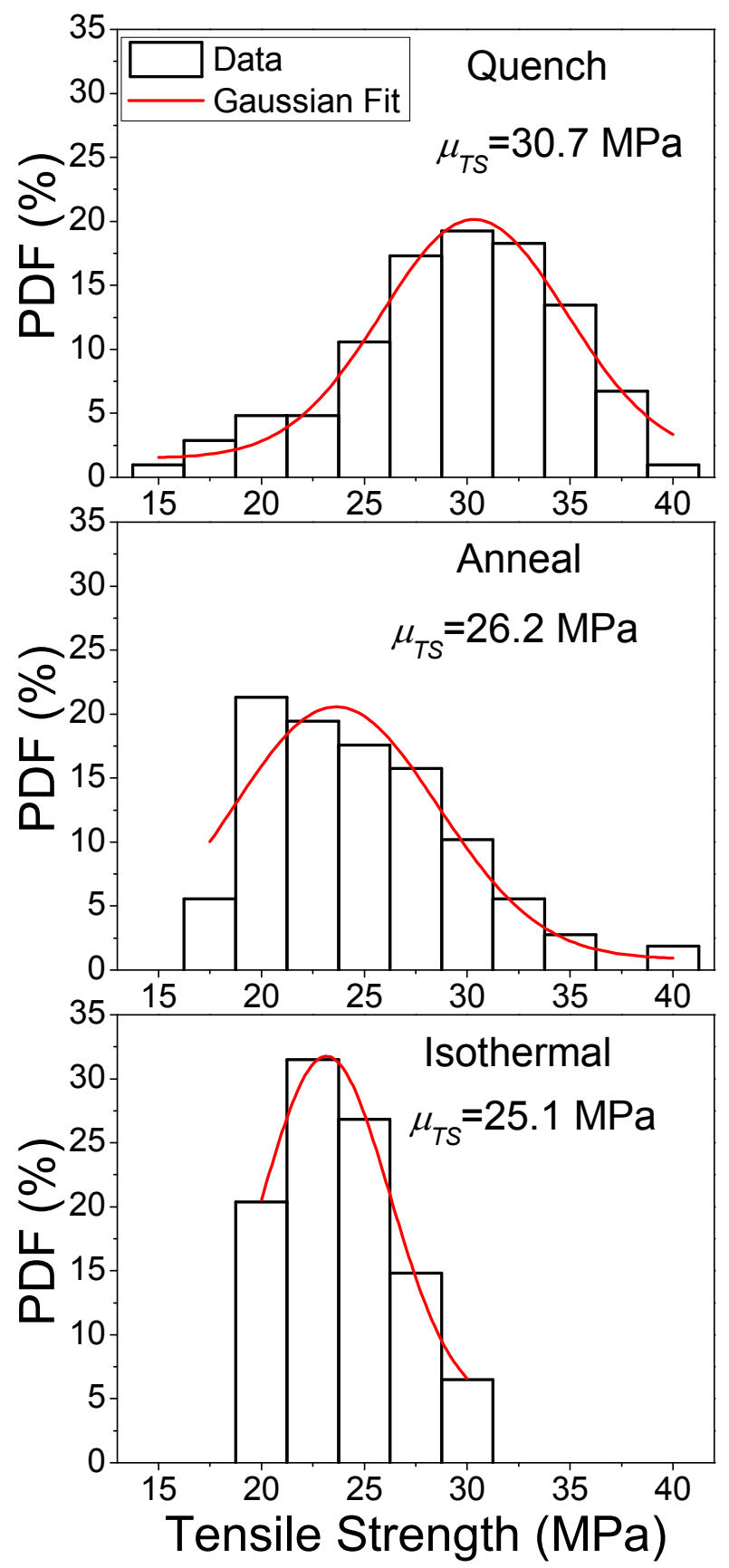

Figure S5. Probability density functions of the tensile strength for HDPE-151K (from top to bottom: quenched, annealed, and isothermally crystallized samples). The average tensile strength value is indicated in the inset of the figure. 


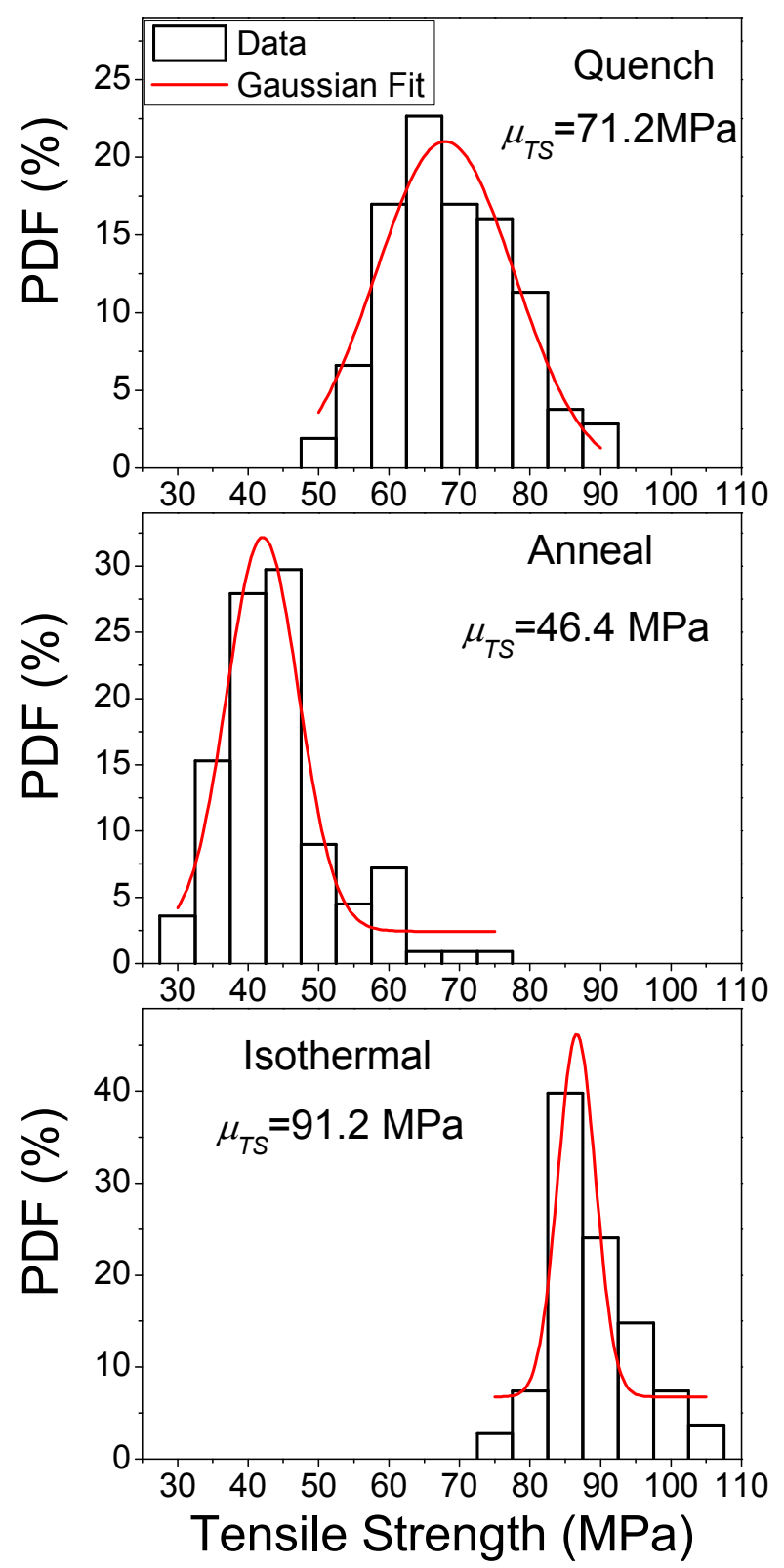

Figure S6. Probability density functions of the tensile strength for HDPE-932K (from top to bottom: quenched, annealed, and isothermally crystallized samples). The average tensile strength value is indicated in the inset of the figure. 